\title{
MODAL LOGIC VS. ONTOLOGICAL ARGUMENT
}

\author{
ANDRZEJ BILAT \\ College of Enterprise and Administration in Lublin
}

\begin{abstract}
The contemporary versions of the ontological argument that originated from Charles Hartshorne are formalized proofs (in the metalogical sense of the word) based on unique modal theories. The simplest well-known theory of this kind arises from the $B$ system of modal logic by adding two extra-logical axioms: (AA) "If the perfect being exists, then it necessarily exists" (Anselm's Axiom) and (AL) "It is possible that the perfect being exists" (Leibniz's Axiom). In the paper a similar argument is presented, however none of the systems of modal logic is relevant to it. Its only premises are the axiom (AA) and, instead of $(\mathrm{AL})$, the new axiom (AN): "If the perfect being doesn't exist, it necessarily doesn't". The main goal of the work is to prove that (AN) is no more controversial than (AA) and - in consequence - the whole strength of the modal ontological argument lies in the set of its extra-logical premises. In order to do that, three arguments are formulated: ontological, "cosmological" and metalogical.
\end{abstract}

1. Pursuant to the definition presented by Boethius and Anselm, the perfect being (i.e. the greatest being or simply God) is something than which nothing greater can be thought. This definition is the key premise in the original argumentation of Anselm supporting the thesis about the existence of the perfect being. ${ }^{1}$ This is a popular summary of the argumentation: "Now that which is such that nothing greater can be conceived cannot exist only in the intellect. For indeed, to exist in reality is greater than to exist in the intellect only. If, then, that which is

\footnotetext{
${ }^{1}$ It is well known that both different versions of this argumentation and its criticism (started by Gaunilon) have had many centuries of tradition. This tradition will not be further discussed here.
} 
such that one can conceive nothing greater exists only in intellect, that than which is nothing greater can be conceived is that than is something greater can be conceived, which is contradictory. The being than which none greater can be conceived then necessarily exists both in the intellect and in reality." (Gilson 1955: 133).

2. One of the assumptions of Anselm's argumentation - expressed in the last sentence of the above mentioned citation - is a thesis according to which if the perfect being exists, it does so out of necessity; formally:

$$
\text { (AA) } \quad p_{0} \rightarrow \mathrm{L} p_{0}
$$

where $p_{0}$ is the propositional constant representing the sentence "The perfect being exists". This premise seems not to be very controversial. It stems from the Boethius-Anselm's definition and an intuitive assumption that a non-contingent being is greater than a contingent being.

The so-called Leibniz's axiom says that the existence of the perfect being is possible:

$$
\text { (AL) } \quad \mathrm{M} p_{0}
$$

Leibniz was the first philosopher who considered this premise crucial in the ontological proof and analysed it in detail (including an argument attempt). The AL principle is not as obvious as the AA one, but it seems - at least at first sight - not to be very strong: in order to prove the possibility that a given being exists, it is enough to prove its cohesive theory (in this case - a cohesive theory of the perfect being; this issue will not be further discussed here).

3. I will now present a proof that is a possibly simpler version of the ontological argument in Hartshorn's style. This version is formulated within the framework of the B system of the modal logic (a sub-system of the popular S5 system of the modal logic) supported by additional AA and AL axioms.
1. $\mathrm{L}\left(p_{0} \rightarrow \mathrm{L} p_{0}\right)$
Gödel's rule, AA,
2. $\mathrm{M} p_{0} \rightarrow \mathrm{ML} p_{0}$ 1, modal logic: $\mathrm{L}(p \rightarrow q) \rightarrow(\mathrm{M} p \rightarrow \mathrm{M} q)$,
3. $M L p_{0} \rightarrow p_{0}$ B system: ML $p \rightarrow p$,
4. $\mathrm{M} p_{0} \rightarrow p_{0}$
2,3 ,
5. $p_{0}$ 4, AL. 
There are also more complex versions of the modal ontological argument (e.g. Alvin Plantinga's) which involve an advanced ontology of possible worlds. These versions usually employ the S5 modal logic principle that is stronger than the $\mathrm{B}$ one.

4. Irrespective of these differences, usually two sources of the surprising strength of the modal ontological argument are named: a) AL and b) B thesis used in step 3 or a stronger S5 thesis. It is supported by a fragment of the "Ontological argument" entry presented in a popular philosophy dictionary: "This concession is much more dangerous than it looks, since in the modal logic involved, from possibly necessarily $p$, we can derive necessarily p." (Blackburn 1994: 269)

A similar point of view was presented by John L. Mackie, the author of a well-known work on the arguments for and against theism. According to him, AL only looks innocent, while this postulate, taking account of the specific S5 thesis (saying that everything that is possibly necessary is indeed necessary), is in fact a Trojan horse rather than an innocent, insignificant option. As said by Mackie, a change in the basis for reasoning into a relevant system of the modal logic would not allow Plantinga's argumentation to develop (Mackie 1982).

In the context of discussions focused on the modal ontological argument, such opinions emphasise the popularity of a conviction according to which certain specific principles of the modal logic are crucial premises of this argument and that adopting a weak system of this logic, e.g. T or S4 system, blocks the argument. ${ }^{2}$ Further considerations will prove that this conviction is faulty.

5. Let's call a thesis claiming that 'if the perfect being does not exist, it does not exist out of necessity' the axiom of the non-existence of the perfect being (in short: AN); formally:

(AN) $\neg p_{0} \rightarrow \mathrm{L} \neg p_{0}$

Based on this premise, a very simple version of the modal ontological argument can be formulated. This can be done within any system of the modal logic supported by additional AN and AL axioms.

$$
\text { 1. } \neg \mathrm{L} \neg p_{0} \rightarrow p_{0} \quad \text { AN, law of transposition, }
$$

${ }^{2}$ Jerzy Perzanowski presents a similar point of view: "The ontological argument requires a legitimate and careful selection of the basic logic". (Perzanowski 1994: 95) 
$\begin{array}{ll}\text { 2. } \mathrm{M} p_{0} \rightarrow p_{0} & \text { 1, definition: } \mathrm{M} p \equiv \neg \mathrm{L} \neg p, \\ \text { 3. } p_{0} & \text { 2, AL. }\end{array}$

Various formulations of the AN axiom were considered by Hartshorn (1965), Plantinga (1974) and Malcolm (1960). For example Norman Malcolm wrote: "What Anselm has proved is that the notion of contingent existence or of contingent nonexistence cannot have any application to God. His existence must either be logically necessary or logically impossible. [...] If God, a being a greater than which cannot be conceived, does not exist then $\mathrm{He}$ cannot come into existence. [...] Since He cannot come into existence, if He does not exist His existence is impossible." (Malcolm 1960: 49)

In fact, it seems that $\mathrm{AN}$ is not more controversial than AA. It becomes even clearer after taking account of some theoretical contexts typical of such considerations: ontological, "cosmological" and metalogical.

6. The AA content can be expressed in the language of the ontology of possible worlds in the following way: if the perfect being exists in the actual world, it exists in every possible world. The AN content is the following: if the perfect being does not exist in the actual world, it does not exist in any possible world. Both principles follow from a stronger postulate:

(P) The perfect being exists either in every possible world or in none of them. If the perfect being existed only in some of the possible worlds, it would be a contingent being rather than the perfect one. Thus, the $(\mathrm{P})$ principle seems to be an entirely natural explanation of intuitions included both in $\mathrm{AA}$ and $\mathrm{AN}$. If so, $\mathrm{AN}$ is not more controversial than AA.

7. A similar conclusion can be reached taking account of an additional, "cosmological" assumption, according to which the perfect being is the only creator of the world. Let's assume a reality different than the one propagated by AA. Namely, the perfect being exists but it is not necessary. In consequence, the world has been created, although it did not have to be. This outcome does not seem to be logically contradictory, taking account of an intuition according to which the perfect being does not need any other being to exist.

Let's now assume that the reality contradicts the AN postulate, i.e. the perfect being does not exist, although it is possible. In consequence, the world was not created, although it could have been. This seems impossible: a world cannot be created if its potentially sole creator does 
not exist. One way or another, taking the "cosmological" context into account in no way makes the AN postulate more controversial (easier to be refuted) than the AA one.

8. The aim of this point is to use metalogical tools to prove that the argument presented in point 5 is based on significantly weaker assumptions than the modal argument in Hartshorn's style.

Let $\mathrm{Cn}$ be a standard (classical) consequence operation defined on the power set of formulas of the propositional modal logic extended by the $p_{0}$ form. Let's use the following indications:

$$
\begin{aligned}
& \mathrm{TH}=\mathrm{Cn}(\mathrm{B} \cup\{\mathrm{AA}, \mathrm{AL}\}), \\
& \mathrm{TN}=\mathrm{Cn}(\{\mathrm{AL}, \mathrm{AN}\}) .
\end{aligned}
$$

Hence, $\mathrm{TH}$ is a theory created by extending the B system of propositional modal logic with the AA and AL axioms, while TN is a theory created by extending the system of the classical propositional logic with the AL and $\mathrm{AN}$ axioms.

METATHEOREM 1. AN $\in$ TH

PROOF. Based on the reasoning conducted in point 3 , we know that the $p_{0}$ sentence is the TH thesis. This substitution of Scotus's law is also a TH thesis:

$$
p_{0} \rightarrow\left(\neg p_{0} \rightarrow \mathrm{L} \neg p_{0}\right) \in \mathrm{TH} .
$$

So we obtain by Modus Ponens:

$$
\left(\neg p_{0} \rightarrow \mathrm{L} \neg p_{0}\right) \in \text { TH. Q.E.D. }
$$

Since AL and AN are TH theses, TN is a subset of TH. At the same time, TH is not identical to TN: Gödel's rule and B-system axioms are not present in TN. In consequence, the TN theory is essentially weaker than the TH theory:

CONCLUSION $1 . \quad$ TN $\subsetneq$ TH.

METATHEOREM 2. $\quad p_{0} \in \mathrm{TN}$.

PROOF. The proof is very similar to the argument presented in point 5. Q.E.D. CONCLUSION 2. The sentence "The perfect being exists" is a thesis of the modal theory which: a) is a part of a theory which is the standard basis for the modal ontological argument and b) does not contain any specific principles of the modal logic. 
9. Therefore, it has turned out that specific theses of the modal logic i.e. B or S5 - are not essential in the modal version of the ontological argument. In other words, the whole strength of the modal ontological argument lies in its premises rather than in its logical principles. This outcome undermines a quite popular conviction according to which such principles are indispensable.

Taking account of the persuasive strength of the AA and AN assumptions and (indicated in point 4) interpretations in which it is Leibniz's axiom (and specific principles of some of the modal logic systems) to be treated as the Trojan horse of the ontological argument, a question arises whether the main source of strength of this argument indeed comes down to this axiom.

According to Leibniz, the basis for the modal ontological argument is the conditional thesis saying that if the perfect being is possible, it exists (line 2 in the proof presented in point 5). Taking account of the fact that it was deduced from the obvious law of the classical logic (law of transposition) and from the definition of the possibility operator, it could be adopted instead of AN as a premise of this argument. Similarly to AA and AN, it has a character of a semantic postulate for the notion "perfect being”. Together with Leibniz's Axiom it creates a specific TN' theory built of two axioms:

$$
\mathrm{TN}^{\prime}=\mathrm{Cn}\left(\left\{\mathrm{M} p_{0} \rightarrow p_{0}, \mathrm{M} p_{0}\right\}\right) .
$$

(This theory is equivalent of TN on the basis of a standard definition of the operator M.) Using these axioms to generate the thesis on the existence of the perfect being is the most trivial deduction of all. This, however, is under one condition. Namely, both premises, i.e. the whole theory, need to be accepted jointly.

The final diagnosis of the source of the quite surprising strength of the modal ontological argument is the following. No specific laws of the modal logic can constitute this source. In other words, selecting the modal logic does not matter in the argument construction. This source is not Leibniz's axiom (AL) or Anselm's principle (AA) or the condition for the non-existence of the perfect being (AN) either - if every of these postulates is taken into consideration separately. The Trojan horse for this argument is Leibniz's theory of perfection, TN'. 


\section{BIBLIOGRAPHY}

Blackburn, S. 1994. The Oxford Dictionary of Philosophy (Oxford: Oxford University Press)

Gilson, E. 1955. History of Christian Philosophy in the Middle Ages (New York: Random House)

Hartshorn, C. 1965. Anselm's Discovery. A Re-Examination of the Ontological Proof for God's Existence (La Salle: Open Court)

Mackie, J. L. 1982. The Miracle of Theism: Arguments for and Against the Existence of God (Oxford: Oxford University Press)

Malcolm, N. 1960. "Anselm's Ontological Arguments", The Philosophical Review, Vol. 69, No. 1, pp. 41-62

Perzanowski, J. 1995. „O wskazanych przez Ch. Hartshorne’a modalnych krokach w dowodzie ontologicznym św. Anzelma", in: Filozofia/logika - filozofia logiczna 1994, eds. J. Perzanowski, A. Pietruszczak, C. Mordka (Toruń: UMK), pp. 77-96 (in Polish)

Plantinga, A. 1974. The Nature of Necessity (Oxford: Oxford University Press) 